\title{
ESPACES À CONNEXIONS AFFINES ET RIEMANNIENNES SYMÉTRIQUES
}

\author{
SHÔSHICHI KOBAYASHI
}

Le but de la présente note est de clarifier la notion de variété riemanienne ou affine symétrique (et plus généralement, localement réductive) du point de vue de la théorie de connexions dans les espaces fibrés. Nous donnerons quelques caractérisations des espaces localement symétriques, ou réductives (Th. 1.2 et 3 ). On trouvera la définition de dérivation covariante de tenseurs de courbure et de torsion dans la terminologie de la théorie de connexions dans les espaces fibrés. Cette définition peut être généralisée à la dérivation covariante d'un tenseur quelconque. Les résultats concernant la dérivation covariante seront publiés ailleurs. Enfin on retrouvera des résultats de BorelLichnerowicz. Même si l'on ne trouvera pas de résultats nouveaux dans cette note, on comprendra mieux les espaces symétriques par la théorie de connexion de Cartan.

Je tiens à remercier ici à mon ami K. Nomizu, auquel je dois de nombreuses améliorations dans l'exposition de cette note.

\section{Connexion de Cartan}

Soit $E$ une variété fibrée de base $V$, de fibre $F$ et de groupe structural de Lie $G$. $E$ est dite soudée à $V$ [4], si les conditions suivantes sont satisfaites.

(1) $G$ opère transitivement sur $F$; c'est-à-dire $F$ peut être identifiée à l'espace homogéne $G / G^{\prime}$, où $G^{\prime}$ est le groupe d'isotropie en un point $O$ de $F$.

(2) $\operatorname{dim} F=\operatorname{dim} V$.

(3) Le groupe structural $G$ de la variété fibrée $E$ peut être réduit jusqu'à $G^{\prime}$; autrement dit, $E$ admet une section que nous noterons par $\sigma$. Quand la variété $E$ est considérée comme la variété fibrée à groupe structural $G^{\prime}$, elle sera désignée par $E^{\prime}$.

(4) Deux variétés fibrées $T(V)$ et $T_{F}(E)$ de base $V$ sont équivalentes,

Received March 1, 1955. 
où $T(V)$ est l'espace des vecteurs tangents à $V$ et $T_{F}(E)$ est l'espace des vecteurs tangents à $F_{x}$ en $\sigma(x), x$ parcourant dans $V$.

Soit $H$ (resp. $H^{\prime}$ ) l'espace fibré principal associé à $E$ (resp. $E^{\prime}$ ). Le groupe structural de $H$ (resp. $H^{\prime}$ ) est $G$ (resp. $G^{\prime}$ ) ; et $H^{\prime}$ peut être considérée comme une sous-variété de $H$.

Une connexion infinitésimale dans $H$ est définie par une forme différentielle linéaire $\widetilde{\omega}$ sur $H$ satisfaisant aux conditions suivantes:

(c. 1). Les valeurs de $\widetilde{\omega}$ sont dans $g$, algèbre de Lie de $G$.

(c. 2). $\widetilde{\omega}(u \cdot \bar{s})=s^{-1} \bar{s}$ pour tout vecteur $\bar{s}$ tangent à $G$ au point $s$ et pour tout point $u$ de $H$.

(c. 3). $\widetilde{\omega}(\bar{u} \cdot s)=s^{-1} \widetilde{\omega}(\bar{u}) s$ pour tout vecteur tangent $\bar{u}$ à $H$ et pour tout point $s$ de $G$.

Soit $\omega$ la restriction de $\widetilde{\omega}$ sur $H^{\prime}$. Si elle satisfait à la condition suivante, la connexion est appelée une connexion de Cartan [4]:

(c. 4). Si $\omega(\bar{u})=0$, alors $\bar{u}$ est le vecteur zéro.

La dernière condition implique que $\omega$ définit un parallélisme absolu dans $H^{\prime}$.

Pour que la connexion de Cartan définie par $\omega$ soit complète, il faut et il suffit que tout champ de vecteurs $W$ sur $H^{\prime}$ tel que $\omega(W)$ soit une constante engendre un groupe de transformations à 1-paramètre global [6].

\section{Connexion affine [3]}

Soit $V$ une variété différentiable réelle à $n$-dimensions. Nous désignerons par $T_{x}(V)$ l'ensemble de tous les vecteurs tangents à $V$ en un point $x$ de $V$. Soit $F$ un espace vectoriel réel à $n$-dimensions et $G_{x}^{\prime}$ l'ensemble de toutes les applications linéaires non-singulières de $F$ sur $T_{x}(V)$. $G_{x}^{\prime}$ est isomorphe au groupe linéaire $G L(n, R)$. Désignons par $H^{\prime}$ l'ensemble $\bigcup_{x \in V^{\prime}} G_{x}^{\prime}$.

Soit $G$ le groupe des transformations affines de $F$ avec les translations et $G^{\prime}$ le groupe des transformations affines centrales de $F$; c'est-à-dire $G^{\prime}=G L(n, R)$. On a

$$
G / G^{\prime}=F
$$

$T(V)$ est une variété fibrée de base $V$, de fibre $F$ et de groupe structural $G$ et elle est soudée à $V$. Considérons $T(V)$ comme une variété fibrée de 
groupe structural $G^{\prime}$. Alors l'espace fibré principal associé à $T(V)$ est $H^{\prime}$.

Soit $\left(b_{1}, \ldots, b_{n}\right)$ une base de l'espace vectoriel $F$ et $u$ un élément de $G_{x}^{\prime}$. Alors $\left(e_{1}(x), \ldots, e_{n}(x)\right)$, où $e_{\alpha}(x)=u\left(b_{\alpha}\right)$ pour $\alpha=1, \ldots, n$, est appelé un repère au point $x$.

Une section locale de la variété fibrée principale $H^{\prime}$ est un ensemble de champs de vecteurs locaux $\left(e_{1}, \ldots, e_{n}\right)$ sur $V$ tels que $\left(e_{1}(x), \ldots, e_{n}(x)\right)$ soit un repère en $x$. Supposons que les $e_{1}, \ldots, e_{n}$ sont définis sur un ensemble ouvert $U$ de $V$, où un système de coordonnées $x^{1}, \ldots, x^{n}$ est valable. Si $\widetilde{\theta}^{1}, \ldots, \widetilde{\theta}^{n}$ sont les formes de Pfaff sur $U$ satisfaisant aux conditions

$$
\tilde{\theta}^{\alpha}\left(e_{\beta}\right)=\delta_{\beta}^{\alpha} \quad \alpha, \beta=1, \ldots, n ;
$$

alors, au moyen de coordonnées locaux, elles sont exprimées comme suivant: ${ }^{*}$

$$
\tilde{\theta}^{\alpha}=A_{\beta}^{\alpha} d x^{\beta} \text {, où } A_{\beta}^{\alpha} \text { sont des fonctions définies sur } U \text {. }
$$

Evidemment la matrice $\left(A_{\beta}^{\alpha}\right)$ est non-singulière. Si $\left(B_{\beta}^{\alpha}\right)$ est la matrice inverse de $\left(A_{\beta}^{\alpha}\right)$, on a

$$
e_{\alpha}=B_{\alpha}^{\beta}\left(\partial / \partial x^{\beta}\right)
$$

Tout point de $G_{x}^{\prime}$, c'est-à-dire tout repère au point $x$, peut être exprimé par les vecteurs suivants :

$$
Y_{\alpha}^{\beta} B_{\beta}^{\Upsilon}\left(\partial / \partial x^{\curlyvee}\right) \quad \alpha=1, \ldots, n,
$$

où $\left(Y_{\beta}^{\alpha}\right)$ est une matrice non-singulière. Nous adoptons les $x^{\alpha}, Y_{\beta}^{\alpha}$ comme un système de coordonnées dans $\pi^{-1}(U)$, où $\pi$ est la projection de $H^{\prime}$ sur $V$.

Supposons qu'une connexion affine est définie sur $V$ par les symboles de Christoffel $\Gamma_{\sigma \tau}^{\mu}$ et considérons les formes de Pfaff sur $\pi^{-1}(U)$ :

$$
\begin{gathered}
\theta^{\alpha}=X_{\beta}^{\alpha} A_{\gamma}^{\beta} d x^{\gamma} \text {, où }\left(X_{\beta}^{\alpha}\right) \text { est la matrice inverse de }\left(Y_{\beta}^{\alpha}\right) . \\
\omega_{\beta}^{\alpha}=X_{\lambda}^{\alpha} A_{\mu}^{\lambda} d\left(B_{\nu}^{\mu} Y_{\beta}^{\nu}\right)+X_{\lambda}^{\alpha} A_{\mu}^{\lambda} \Gamma_{\sigma \tau}^{\mu} d x^{\tau} B_{\nu}^{\alpha} Y_{\beta}^{\nu} .
\end{gathered}
$$

On voit aisément que les formes $\theta^{\alpha}, \omega_{\beta}^{\alpha}$ sont indépendantes du choix de coordonnées et qu'elles peuvent être considérées comme les formes définies sur la variété $H^{\prime}$ toute entière. Elles définissent une connexion de Cartan dans l'espace fibré $T(V)$ soudé à $V ; \omega_{\beta}^{\alpha}$ appartiennent à l'algèbre de Lie de $G^{\prime}$ et $\theta^{\alpha}$ à l'algèbre de Lie du sous-groupe de $G$ formé par les translations de $F$.

\footnotetext{
* Les mêmes indices figurant dans un terme indiquent la sommation.
} 
Soit $T_{\beta \delta}^{\alpha}$ le tenseur de torsion et $R_{\beta \gamma \delta}^{\alpha}$ celui de courbure par rapport aux coordonnées $x^{1}, \ldots, x^{n}$. Posons

$$
X_{\beta}^{\prime \alpha}=X_{\Upsilon}^{\alpha} A_{\beta}^{\Upsilon}, \quad Y_{\beta}^{\prime \alpha}=B_{\gamma}^{\alpha} Y_{\beta}^{\gamma} .
$$

Comme le montre un calcul simple, on a

$$
\begin{gathered}
d \theta^{\alpha}=-\omega_{\beta}^{\alpha} \theta^{\beta}-T_{\beta \gamma}^{\prime \alpha} \theta^{\beta} \wedge \theta^{\gamma}, \quad \text { où } T_{\beta \gamma}^{\prime \alpha}=X_{\lambda}^{\prime \alpha} T_{\mu \nu}^{\lambda} Y_{\beta}^{\prime \mu} Y_{\gamma}^{\prime \nu} . \\
d \omega_{\beta}^{\alpha}=-\omega_{\gamma \wedge}^{\alpha} \omega_{\beta}^{\gamma}-R_{\beta \gamma \delta}^{\prime \alpha} \theta_{\wedge}^{\gamma} \theta^{\delta}, \quad \text { où } R_{\beta \curlyvee \delta}^{\prime \alpha}=X_{\kappa}^{\prime \alpha} R_{\lambda \mu \nu}^{\alpha} Y_{\beta}^{\prime \lambda} Y_{\gamma}^{\prime \mu} Y_{\delta}^{\prime \nu} .
\end{gathered}
$$

Notons que les $T_{\beta \delta}^{\alpha}$ et les $R_{\beta \gamma \delta}^{\alpha}$ sont les composantes de tenseur de torsion et de celui de courbure respectivement par rapport aux coordonnées locaux $x^{1}, \ldots, x^{n}$ et que, par contre, les $T_{\beta \gamma}^{\prime \alpha}$ et les $R_{\beta \gamma \delta}^{\prime \alpha}$ sont des fonctions définies sur $H^{\prime}$ toute entière.

\section{Connexion riemannienne [3]}

Supposons que $V$ est une variété riemannienne à métrique $d s^{2}$. Chaque espace tangent $T_{x}(V)$ est muni de la structure de l'espace euclidien à $n$-dimensions. Soit $G$ le groupe de mouvement opérant sur l'espace euclidien $F \grave{a}$ $n$-dimensions et $G^{\prime}$ le groupe d'isotropie à l'origine de $F$. L'espace fibré $T(V)$ de base $V$, de fibre $F$ et de groupe structural $G$ est soudé à $V$ dans le sens défini dans $\S 1$. Considérons $T(V)$ comme l'espace fibré de groupe structural $G^{\prime}$, ce qui est possible, et soit $H^{\prime}$ l'espace fibré principal associé à $T(V)$.

Soit $\left(e_{1}, \ldots, e_{n}\right)$ un champ de repères orthonormés défini sur un ensemble ouvert $U$ de $V$. Si $\widetilde{\theta}^{1}, \ldots, \widetilde{\theta}^{n}$ sont les formes différentielles définies par (2.1), on a

$$
d s^{2}=\widetilde{\theta}^{\alpha} \widetilde{\theta}^{\alpha}
$$

Si l'on définit $\left(A_{\beta}^{\alpha}\right)$ et $\left(B_{\beta}^{\alpha}\right)$ comme dans $\S 2$, tout repère orthonormé en un point $x$ est de la forme

$$
Y_{\alpha}^{\beta} B_{\beta}^{\gamma}\left(\partial / \partial x^{\gamma}\right)
$$

où $\left(Y_{\beta}^{\alpha}\right)$ est une matrice orthogonle. Les $x^{\alpha}, Y_{\beta}^{\alpha}$ seront pris comme un système de coordonnées locaux de $H^{\prime}$, même s'ils ne sont pas indépendants.

Si l'on définit les formes $\theta^{\alpha}$, $\omega_{\beta}^{\alpha}$ sur $H^{\prime}$ de la meme manière que (2.5) et (2.6) respectivement, on a 


$$
\begin{gathered}
\omega_{\beta}^{\alpha}=-\omega_{\alpha}^{\beta} \\
d \theta^{\alpha}=-\omega_{\beta \wedge}^{\alpha} \theta^{\beta} . \\
d \omega_{\beta}^{\alpha}=-\omega_{\curlyvee \wedge}^{\alpha} \omega_{\beta}^{\gamma}-R_{\beta \curlyvee \delta}^{\prime \alpha} \theta_{\wedge}^{\gamma} \theta^{\delta} .
\end{gathered}
$$

Les formes $\omega_{\beta}^{\alpha}$ appartiennent à l'algèbre de Lie du groupe orthogonal $G^{\prime}$ et les formes $\theta^{\alpha}$ à l'algèbre de Lie du sous-groupe de $G$ formé par les translations de $F$. Les $\theta^{\alpha}$ et $\omega_{\beta}^{\alpha}$ définissent une connexion de Cartan dans $T(V)$.

Remarque: Dans le cas riemannien, les formes $\theta^{\alpha}$ et $\omega_{\beta}^{\alpha},(\alpha<\beta)$, sont linéairement indépendantes et définissent un parallélisme absolu sur $H^{\prime}$.

\section{Espace localement symétrique}

Revenons à la connexion affine et employons les mêmes notations que dans $\S 2$. Soient $W_{1}, \ldots, W_{n}$ les champs de vecteurs sur $H^{\prime}$ satisfaisant aux conditions suivantes:

$$
\begin{aligned}
& \theta^{\alpha}\left(W_{\beta}\right)=\delta_{\beta}^{\alpha}, \\
& \omega_{\gamma}^{\alpha}\left(W_{\beta}\right)=0 .
\end{aligned}
$$

Les expressions explicites de $W_{\alpha}$ sont données par

$$
W_{\alpha}=Y_{\alpha}^{\prime \beta}\left(\partial / \partial x^{\beta}-\Gamma_{\gamma \beta}^{\delta} Y_{\varepsilon}^{\prime \gamma} \cdot \partial / \partial Y_{\varepsilon}^{\prime \delta}\right)
$$

Les $T_{\beta \gamma}^{\prime \alpha}$ et $R_{\beta \gamma \delta}^{\prime \alpha}$ sont des fonctions numériques définies sur la variété $H^{\prime}$ toute entière. En appliquant les transformations infinitésimales $W_{\varepsilon}$ aux fonctions $T_{\beta \gamma}^{\prime \alpha}$ et $R_{\beta \gamma \delta}^{\prime \alpha}$, on obtient

$$
\begin{gathered}
W_{\varepsilon}\left(T_{\beta \gamma}^{\prime \alpha}\right)=X_{\lambda}^{\prime \alpha} T_{\mu \nu ; \rho}^{\lambda} Y_{\beta}^{\prime \mu} Y_{\gamma}^{\prime \nu} Y_{\varepsilon}^{\prime \rho}, \\
W_{\varepsilon}\left(R_{i \gamma \delta}^{\prime \alpha}\right)=X_{\Lambda}^{\prime \alpha} R_{\mu \nu \rho ; \tau}^{\lambda} Y_{\beta}^{\prime \mu} Y_{\gamma}^{\prime \nu} Y_{\delta}^{\prime \rho} Y_{\varepsilon}^{\prime \tau},
\end{gathered}
$$

où $T_{\mu \nu ; \rho}^{\lambda}$ et $R_{\mu \nu \rho ; \tau}^{\lambda}$ sont les dérivées covariantes de tenseurs de torsion et de courbure respectivement.

Ainsi nous avons démontré le

THÉORÈME 1. Soit $V$ une variété à connexion affine et $H^{\prime}$ l'espace fibré principal des repères sur $V$. Soit $\theta^{\alpha}$ et $\omega_{\beta}^{\alpha}$ les formes de Pfaff sur $H^{\prime}$ definissant la connexion affine. Alors les dérivées covariantes de tenseurs de torsion et de courbure s'annulent, si et seulement si 


$$
W_{\varepsilon}\left(T_{\beta \gamma}^{\prime \alpha}\right)=0 \text {, }
$$

$$
W_{\varepsilon}\left(R_{\beta \curlyvee \delta}^{\prime \alpha}\right)=0
$$

pour tout champ de vecteurs $W_{\varepsilon}$ sur $H^{\prime}$ satisfaisant aux conditions (4.1) et (4.2).

Définition: Une variété $V$ munie d'une connexion affine est dite localement réductive, si elle satisfait aux conditions (4.6) et (4.7). Elle est localement symétrique, si elle satisfait à la condition (4.7) et si le tenseur de torsion s'annule.

Remarque: Il ne faut pas confondre "localement symétrique" et "sans torsion"; $V$ est localement symétrique, si la connexion est sans torsion et si les dérivées covariantes de tenseur de courbure s'annulent.

Ce que nous avons obtenu est aussi valable pour une variété riemannienne.

Corollaire. Soit $V$ une variété riemannienne et $H^{\prime}$ l'espace fibré des repéres orthonormés sur $V$. Supposons que les formes $\theta^{\alpha}$ et $\omega_{\beta}^{\alpha}$ sur $H^{\prime}$ definissant la connexion riemannienne. Alors les dérivées covariantes de tenseur de courbure s'annulent, si et seulement si

$$
W_{\varepsilon}\left(R_{\beta \gamma \delta}^{\prime \alpha}\right)=0,
$$

ou les $W_{\varepsilon}$ sont définies par (4.1) et (4.2).

Une variété riemannienne $V$ satisfaisant à la condition (4.8) est localement symétrique.

On peut trouver dans [7] l'interprétation géométrique de notions: "localement réductive" et "localement symétrique".

Remarque: Si l'on veut éviter l'emploie d'indices, on n'a qu'à considérer la forme de Pfaff $\omega$ à valeurs dans l'algèbre de Lie de $G$, qui définit la connexion affine (ou riemannienne). Alors l'équation de structure s'écrit [1]

$$
d \omega=-[\omega, \omega] / 2+\Omega,
$$

où $\Omega$ est une forme de degré 2 à valeurs dans l'algèbre de Lie de $G$ et s'appelle la forme de courbure. Considérons la décomposition de la forme $\omega$ dans les deux forme $\theta$ et $\omega^{\prime}$ :

$$
\omega=\theta+\omega^{\prime},
$$


où $\omega^{\prime}$ (resp. $\theta$ ) est une forme à valeurs dans l'algèbre de Lie de $G^{\prime}$ (resp. du sous-groupe de $G$ formé par les translations de $F$ ). Alors la variété $V$ à connexion affine (ou riemannienne) est localement réductive si et seulement si

$$
W_{1}\left(\Omega\left(W_{2}, W_{3}\right)\right)=0
$$

pour tout choix de transformations infinitésimales $W_{1}, W_{2}, W_{3}$ sur $H^{\prime}$ satisfaisant à la condition suivante:

$$
\theta\left(W_{i}\right)=\text { constante, } \quad \omega^{\prime}\left(W_{i}\right)=0 \quad \text { pour } i=1,2,3 \text {. }
$$

Nous expliquons (4.11); $\Omega$ étant une 2-forme sur $H^{\prime}, \Omega\left(W_{2}, W_{3}\right)$ est une fonction sur $H^{\prime}$, et on applique une transformation infinitésimale $W_{1}$ à la fonction $\Omega\left(W_{2}, W_{3}\right)$.

\section{Réduction de groupe structural}

Soit $V$ une variété à connexion affine (ou riemannienne) et $H^{\prime}$ l'espace fibré principal des repères (ou repères orthonormés) sur $V$. Un vecteur $\bar{u}$ tangent à $H^{\prime}$ est dit horizontal [1], si

$$
\begin{gathered}
\theta^{\alpha}(\bar{u})=\text { constante, } \\
\omega_{\beta}^{\alpha}(\bar{u})=0 .
\end{gathered}
$$

Une courbe dans $H^{\prime}$ est horizontale, si tout vecteur tangent à cette courbe est horizontal. Il est évident qu'un vecteur tangent $\bar{u}$ est horizontal, si et seulement si $\bar{u}$ est contenu dans l'espace des combinaisons linéaires des $W_{1}, \ldots, W_{n}$ à coefficients constants réels.

Prenons un point arbitraire $u_{0}$ dans $H^{\prime}$ et soit $H^{\circ}$ l'ensemble des points de $H^{\prime}$ qui peuvent être joints à $u_{0}$ par des courbes horizontaux. $H^{\circ}$ est une variété fibrée principale de base $V[1]$, dont le groupe structural est le groupe d'holonomie homogène de la connexion affine envisagée.

THÉORÈme 2. Pour que $V$ est localement réductive, il faut et il suffit que les fonctions $T_{\beta \gamma}^{\prime \alpha}$ et $R_{\beta \gamma \delta}^{\prime \alpha}$ sont toutes des constantes sur $H^{\circ}$.

Démonstration: Si $V$ est localement réductive, on a

$$
\begin{aligned}
& \bar{u}\left(T_{\beta \gamma}^{\prime \alpha}\right)=0, \\
& \bar{u}\left(R_{\beta \gamma \delta}^{\prime \alpha}\right)=0
\end{aligned}
$$


pour tout vecteur tangent horizontal $\bar{u}$, et inversement. Ce qui montre notre théorème.

\section{Equations de structure}

Considérons la réstriction des formes $\theta^{\alpha}$ et $\omega_{\beta}^{\alpha}$ sur $H^{\circ}$. Les formes $\theta^{\alpha}$ sont encore linéairement indépendantes sur $H^{\circ}$; mais les formes $\omega_{\beta}^{\alpha}$ restreintes sur $H^{\circ}$ ne sont plus linéairement indépendantes en général et il existe des constantes $c_{\alpha}^{i \beta}(i=1, \ldots, r ; \alpha, \beta=1, \ldots, n)$ telles que les formes

$$
\omega^{i}=c_{\alpha}^{i \beta} \omega_{\beta}^{\alpha}(i=1, \ldots, r)
$$

sont linéairement indépendantes sur $H^{\circ}$ et que $r$ est égal à la dimension du groupe d'holonomie homogène. Désignons toujours par les mêmes lettres $\theta^{\alpha}$, $\omega^{i}$ les formes $\theta^{\alpha}, \omega^{i}$ restreintes sur $H^{\circ}$. Elles définissent un parallélisme absolu sur $H^{\circ}$.

Les formules (2.8) et (2.9) nous conduisent aux équations de structure de Cartan dans $H^{\circ}$ :

$$
\begin{aligned}
& d \theta^{\alpha}=a_{i \beta}^{\alpha} \omega^{i} \wedge \theta^{\beta}+b_{\beta \gamma}^{\alpha} \theta^{\beta} \wedge \theta^{\Upsilon}, \\
& d \omega^{i}=p_{j k}^{i} \omega^{j} \wedge \omega^{k}+q_{\beta \gamma}^{i} \theta^{\beta}{ }_{\wedge} \theta^{\Upsilon},
\end{aligned}
$$

où les $a, b, p, q$ sont des fonctions sur $H^{\circ}$ et, si les fonctions $T_{\beta \gamma}^{\prime \alpha}, R_{\beta \gamma \delta}^{\prime \alpha}$ sont constantes sur $H^{\circ}$, elles sont aussi constantes et vice versa. Ainsi le théoréme 2 entraine le

TheORÈme 3. Si $V$ est localement réductive, alors les équations de structure de Cartan dans $H^{\circ}$ sont les équations de Maurer-Cartan; c'est-à-dire les fonctions $a_{i \beta}^{\alpha}, b_{\beta \gamma}^{\alpha}, p_{j k}^{i}$ et $q_{\beta \gamma}^{i}$ dans (6.2) et (6.3) sont des constantes, et inversement.

\section{Groupe de transformations affines et isométriques}

Supposons que les $\theta^{\alpha}$ et $\omega_{\beta}^{\alpha}$ sont les formes sur $H^{\circ}$ définissant la connexion affine (ou riemannienne). Considérons une transformation différentiable $\varphi$ de $H^{\circ}$ telle que

$$
\begin{aligned}
& \varphi^{*}\left(\theta^{\beta}\right)=\theta^{\alpha}, \\
& \varphi^{*}\left(\omega^{i}\right)=\omega^{i},
\end{aligned}
$$


où $\varphi^{*}$ est l'application induite canoniquement de $\varphi$. J'ai démontré [5] que l'ensemble de toutes les transformations $\varphi$ de $H^{\circ}$ satisfaisant aux conditions (7.1) et (7.2) forme un groupe de Lie, que nous noterons par $A\left(H^{\circ}\right)$ (ou $I\left(H^{\circ}\right)$ dans le cas riemanien). Soit $u_{0}$ un point de $H^{\circ}$. Alors l'application $\varphi \rightarrow \varphi\left(u_{0}\right)$ de $A\left(H^{\circ}\right)$ (ou $I\left(H^{\circ}\right)$ ) dans $H^{\circ}$ est biunivoque et l'image de cette application est une sous-variété fermée régulière (non nécessairement connexe) de $H^{\circ}$. Par ce plongement du groupe $A\left(H^{\circ}\right)$ (ou $I\left(H^{\circ}\right)$ ) dans $H^{\circ}$, les paramètres s'introduisent naturellement dans $A\left(H^{\circ}\right)$ ( ou $I\left(H^{\circ}\right)$ ) [5].

Remarque. Toute transformation $\varphi$ de $H^{\circ}$ satisfaisant aux conditions (7.1) et (7.2) est induite d'une transformation $\bar{\varphi}$ de $V$ qui laisse invariante la connexion affine au sens classique (dans le cas riemannien $\bar{\varphi}$ est une transformation isométrique de $V$ ). Donc $A\left(H^{\circ}\right)$ (ou $I\left(H^{\circ}\right)$ ) est un sous-groupe du groupe de toutes les transformations de $V$ qui laissent invariante la connexion affine (ou de toutes les transformations isométriques de $V$ ). Le sous-groupe d'isotropie de $A\left(H^{\circ}\right)$ (ou $I\left(H^{\circ}\right)$ ) à chaque point de $V$ est contenu dans le groupe d'holonomie homogène. Si l'on veut le groupe de toutes les transformations affines (ou isométriques) de $V$, il faut considérer $A\left(H^{\prime}\right)$ (ou $I\left(H^{\prime}\right)$ ), dont la définition sera évidente. Par exemple, supposons que $V$ est l'espace euclidien ordinaire. Alors $I\left(H^{\prime}\right)$ est le groupe des mouvements de $V$. Puisque le groupe d'holonomie homogène de l'espace euclidien $V$ se réduise à l'élément neutre, $H^{\circ}$ n'est autre chose que $V$, et $I\left(H^{\circ}\right)$ est le groupe des translations de $V$.

Soit $L^{\prime}$ l'ensemble des champs $Z^{\prime}$ de vecteurs sur $H^{\circ}$ tels que

$$
\begin{aligned}
& \theta^{\alpha}\left(Z^{\prime}\right)=\text { constante, } \\
& \omega^{i}\left(Z^{\prime}\right)=\text { constante. }
\end{aligned}
$$

Si notre connexion de Cartan est complète, alors exp $\left(Z^{\prime}\right)$ est définie sur $H^{\circ}$ toute entière pour tout $Z^{\prime}$ de $L^{\prime}$ et inversement (On désigne par exp $\left(t Z^{\prime}\right)$ le groupe de transformation à 1-paramètre engendré par une transformation infinitésimale $Z^{\prime}, t$ étant le paramètre (Cf. C. Chevalley, Theory of Lie groups)).

Soit $L$ l'ensemble des champs $Z$ de vecteurs sur $H^{\circ}$ satisfaisant à

$$
\left[Z, Z^{\prime}\right]=0 \quad \text { pour tout } Z^{\prime} \in L^{\prime} .
$$

Si la connexion est complète, $L$ est l'algèbre de Lie de $A\left(H^{\circ}\right)$ (ou $I\left(H^{\circ}\right)$ ). 
En effet, si $\exp (Z), Z \in L$, est définie pour un point $u_{0}$ de $H^{\circ}$, alors $\exp (Z$ ) est aussi définie pour un point $u^{\prime}=\exp \left(Z^{\prime}\right) u_{0}$, où $Z^{\prime} \in L^{\prime}$ et

$$
\exp (Z) \boldsymbol{u}^{\prime}=\exp \left(\boldsymbol{Z}^{\prime}\right)\left(\exp (\boldsymbol{Z}) \boldsymbol{u}_{0}\right)
$$

Pour tout point $u$ de $H^{\circ}$, il existe $Z_{1}^{\prime}, \ldots, Z_{k}^{\prime} \in L^{\prime}$ tels que

$$
u=\exp \left(Z_{1}^{\prime}\right) \ldots \exp \left(Z_{k}^{\prime}\right) u_{0} .
$$

Donc $\exp (Z)$ peut être définie sur $H^{\circ}$ toute entière et commute avec tout élément $Z^{\prime}$ de $L^{\prime}$ et ainsi appartient à $A\left(H^{\circ}\right)$ (ou $I\left(H^{\circ}\right)$ ). C'est ce qu'il fallait démontrer.

Remarque. On n'a pas utilisé dans cette section la condition que $V$ est localement réductive et les résultats ci-dessus sont valables pour toute connexion de Cartan.

\section{Espace globalement réductive ou symétrique}

Nous montrerons que, si $V$ est une variété à connexion affine localement réductive (ou riemannienne localement symétrique) complète et simplement connexe, alors le groupe $A\left(H^{\circ}\right)$ (ou $I\left(H^{\circ}\right)$ ) coincide avec $H^{\circ}$; c'est-à-dire, $\varphi \rightarrow \varphi\left(u_{0}\right)$ est une application de $A\left(H^{\circ}\right)$ (ou $I\left(H^{\circ}\right)$ ) sur $H^{\circ}$. Puisque $V$ est complète, il suffit de monter que

$$
\operatorname{dim} L=\operatorname{dim} H^{\circ} \text {. }
$$

Pour cela, on n'a qu'à démontrer que, pour tout vecteur $\bar{u}_{0}$ tangent à $H^{\circ}$ en $u_{0}$, il y a un élément $Z$ de $L$ tel que $Z$ est égal à $\bar{u}_{0}$ au point $u_{0}$ :

Rappelons que $H^{\circ}$ est un espace fibré principal dont le groupe structural est le groupe d'holonomie homogène. Donc le groupe d'holonomie opère sur $H^{\circ}$ à droite; et on prolonge $\bar{u}_{0}$ sur la fibre de $H^{\circ}$ contenant $u_{0}$ par la translation de $\bar{u}_{0}$ par le groupe d'holonomie. L'élément $Z \in L$, s'il y en a, doit coincider avec ce prolongement sur la fibre de $H^{\circ}$ contenant $u_{0}$. Soit $u$ un point arbitraire de $H^{\circ}$. Il existe des $Z_{1}^{\prime}, \ldots, Z_{k}^{\prime} \in L^{\prime}$ tels que

$$
u=\exp \left(Z_{k}^{\prime}\right) \ldots \exp \left(Z_{1}^{\prime}\right) u_{0} .
$$

Nous définissons

$$
Z_{u}=\exp \left(Z_{k}^{\prime}\right) \ldots \exp \left(Z_{1}^{\prime}\right) \bar{u}_{0}
$$


et montrerons que le vecteur tangent $Z_{u}$ en $u$ est indépendant du choix de $Z_{1}^{\prime}, \ldots, Z_{k}^{\prime}$. En effet, supposons que

$$
u=\exp \left(-Z_{k+1}^{\prime}\right) \ldots \exp \left(-Z_{k+m}^{\prime}\right) u_{0}
$$

pour $Z_{k+1}^{\prime}, \ldots, Z_{k+m}^{\prime} \in L^{\prime}$.

Alors

$$
u_{0}=\exp \left(Z_{k+m}^{\prime}\right) \ldots \exp \left(Z_{1}^{\prime}\right) u_{0} .
$$

Soit $c_{i}$ une courbe dans $H^{\circ}$, pour $i=1, \ldots, k+m$, définie par

$$
c_{i}(t)=\exp \left(t Z_{i}^{\prime}\right) \quad 0 \leqq t \leqq 1 .
$$

La courbe jointe

$$
c=c_{k+m} \vee \ldots \vee c_{1}
$$

est fermée. Puisque $V$ est simplement connexe, la courbe $c$ est homotope à une courbe dans la fibre de $H^{\circ}$ contenant $u_{0}$. Et sur cette fibre, l'extension de $u_{0}$ était possible et unique. Maintenant, par un raisonnement classique on peut montrer que $Z_{u}$ est indépendant du choix de $Z_{1}^{\prime}, \ldots, Z_{k}^{\prime}$. Ainsi nous avons obtenu un champ de vecteur $Z$ qui appartient évidemment à $L$ et qui est l'extension de $\bar{u}_{0}$.

THÉORÈME 4. Soit $V$ une varièté à connexion affine localement réductive, complète et simplement connexe. Alors le groupe $A\left(H^{\circ}\right)$ coincide avec $H^{\circ}$.

Remarque. Sous la condition du théorème $4, V$ est un espace homogène $A\left(H^{\circ}\right) / H^{\circ}$ et notre connexion affine est la deuxième connexion canonique sur la variété homogène $V$ dans le sens de Nomizu [7].

THÉORÈme 4'. Soit $V$ une variété riemannienne localement symétrique complète et simplement connexe. Alors le groupe $I\left(H^{\circ}\right)$ coincide avec $H^{\circ}$. Par suite, $V$ est globalement symétrique [2].

Remarque i) Soit $A(V)$ (ou $I(V)$ ) le groupe des transformations affines (ou isométriques) de $V$. Si $V$ satisfait à la condition du théorème 4 (ou $4^{\prime}$ ), alors le groupe d'isotropie linéaire (en chaque point de $V$ ) de $A(V)$ (ou $I(V)$ ) contient le groupe d'holonomie homogène. Le théorème 4 (ou $4^{\prime}$ ) montre qu'il existe un sous-groupe de $A(V)$ ou $(I(V))$ dont le groupe d'isotropie linéaire coincide avec le groupe d'holonomie homogène. 
ii) Soit $\pi_{1}\left(H^{\circ}\right)$ (resp. $\pi_{1}(V)$ ) le groupe d'homotopie de dimension 1 de $H^{\circ}$ (resp. de $V$ ). Alors on peut remplacer la condition de "simplement connexe" dans les théorèmes 4 et $4^{\prime}$ par l'hypothèse suivante: l'image de $\pi_{1}\left(H^{\circ}\right)$ dans $\pi_{1}(V)$ par la projection $\pi: H^{\circ} \rightarrow V$ ne contient que l'élément neutre. Cette hypotèse est satisfaite, si et seulement si, ou bien $V$ est simplement connexe, ou bien $\pi_{1}(V)=2$ et $V$ n'est pas orientable.

\section{Exemples}

Soit $R^{n+1}$ l'espace euclidien à $n+1$-dimensions et $S^{n}$ une sphère dans $R^{n+1}$ définie par

$$
S^{n}=\left\{x \in R^{n+1} ; \quad\|x\|=1\right\}
$$

$1^{\text {er }}$ exemple. La sphère $S^{n}$ munie de la métrique riemannienne induite de celle de l'espace euclidien $R^{n+1}$ est riemannienne symétrique (globalement). Le groupe des transformations isométriques $I\left(S^{n}\right)$ est le groupe orthogonal $O(n+1)$ et le groupe d'isotropie de $I\left(S^{n}\right)$ est le groupe orthogonal $O(n)$. Le groupe d'holonomie homogène de la sphère $S^{n}$ est le groupe orthogonal propre $S O(n)$.

$2^{\text {e }}$ exemple. Considérons la transformation $-e: x \rightarrow-x$ de la sphère $S^{n}$. Cette transformation est isométrique. Le groupe $N$ formé par la transformation $-e$ et par la transformation identique $e$ est un sous-groupe invariant de $I\left(S^{n}\right)$. L'espace quotient $S^{n} / N$, munie de la métrique induite de celle de $S^{n}$, est évidemment localement symétrique ; de plus, il est globalement symétrique, parce que le groupe $I\left(S^{n}\right) / N$ opère sur $S^{n} / N$ transitivement. L'espace $S^{n} / N$ est homéomorphe à l'espace projectif à $n$-dimensions, et il est orientable si $n$ est impair; non orientable si $n$ est pair. Cela montre qu'il existe des espaces riemanniens, qui sont globalement symétriques, mais qui ne satisfont pas à la condition dans la remarque ii) après le théorème $4^{\prime}$. Finalement, je signale que le groupe d'holonomie homogène de $S^{n} / N$ est $S O(n)$ ou $O(n)$ selon que $n$ est impair ou pair.

$3^{\text {e }}$ exemple. Je donne un exemple de variété riemanienne complète et localement symétrique, qui n'est pas globalement symétrique. Considérons le corps des quaternioniens, qui sera identifié avec l'espace euclidien à 4-dimensions si nous prenons $\{1, i, j, k\}$ comme une base. Il est bien connu que l'en- 
semble des quaternioniens dont la longueur est l'unité constitue un groupe multiplicatif, qui est considéré comme un sous-groupe du groupe orthogonal $O(4)$. Considérons le groupe formé par $1,-1, i,-i$. I1 n'est pas sous-groupe invariant du groupe $O(4)$. L'espace quotient de la sphère $S^{3}$ par ce groupe discret, muni de la métrique riemannienne induite de celle de $S^{3}$, est localement symétrique et complet, mais n'est pas globalement symétrique. En effet, le groupe d'holonomie de cet espace riemannien est $O(3)$ et si l'espace est globalement symétrique, le groupe d'isotropie du groupe des transformations isométriques doit contenir le groupe d'holonomie homogène. Pour qu'il en soit ainsi, il faut que le groupe formé par $1,-1, i,-i$ soit un sous-groupe invariant du groupe orthogonal $O(4)$. (Le détail de la démonstration sera laissé aux lecteurs.)

Remarque finale. La question de trouver la condition nécessaire et suffisante pour qu'une variété riemannienne localement symétrique soit globalement symétrique, est laissée ouverte.

\section{BIBLIOGRAPHIE}

[1] Ambrose. W.-Singer, I. M.: A theorem on holonomy, Trans. Amer. Math. Soc. 75 (1953), p. 428-443.

[2] Borel, A., Lichnerowicz, A. Espaces riemanniens et hermitiens symétriques, C. R. Acad. Sci. Paris 234 (1952), p. 2332-2334.

[3] Chern, S. S.: Lecture note in Chicago (1952).

[4] Ehresmann, C.: Les connexions infinitésimales dans un espace fibré différentiable, Colloque de Topologie (Bruxeles. 1950).

[5] Kobayashi, S.: Le groupe des transformations qui laissent invariant le parallélisme, Colloque de Topologie (Strasbourg, 1954).

[6] Kobayashi, S.: Espaces à connexion de Cartan complets, Proc. Jap. Acad. 30 (1954), p. 709-710.

[7] Nomizu, K.: Invariant affine connections on homogeneous spaces, Amer. J. Math. 76 (1954), p. 33-65.

\section{University of Washington}

